\title{
FOURIER COEFFICIENTS OF BOUNDED FUNCTIONS ${ }^{1}$
}

\author{
BERNARD FRIEDMAN
}

The results of this paper are divided into two parts. First: inequalities for the Fourier coefficients of any bounded function; second: an approximation theorem for the Fourier development of an arbitrary bounded function.

Inequalities for Fourier coefficients have been discussed in a paper by Professor Szász. ${ }^{2}$ However, his work deals mainly with linear inequalities for complex coefficients. The inequalities to be investigated in this paper are not linear. Nevertheless, they are the best possible, for this reason: given any set of numbers which makes the inequality an equality, there exists a bounded function which has these numbers as its Fourier coefficients.

A simple illustration will clarify this. Let $f(x)$ be a bounded measurable function in $(-\pi, \pi)$ such that $|f(x)| \leqq 1$. The Fourier coefficients of $f(x)$ are given by the formulae

$$
\begin{array}{r}
a_{n}=\frac{1}{\pi} \int_{-\pi}^{\pi} f(x) \cos n x d x, \quad b_{n}=\frac{1}{\pi} \int_{-\pi}^{\pi} f(x) \sin n x d x, \\
n=1,2,3, \cdots .
\end{array}
$$

Then, it is clear that

$$
\begin{aligned}
& \left|a_{n}\right| \leqq \frac{1}{\pi} \int_{-\pi}^{\pi}|f(x)||\cos n x| d x \leqq \frac{1}{\pi} \int_{-\pi}^{\pi}|\cos n x| d x \\
& \left|b_{n}\right| \leqq \frac{1}{\pi} \int_{-\pi}^{\pi}|f(x)||\sin n x| d x \leqq \frac{1}{\pi} \int_{-\pi}^{\pi}|\sin n x| d x
\end{aligned}
$$

since $|f(x)| \leqq 1$.

Since the cosine is negative in the intervals $(-\pi,-\pi / 2)$ and $(\pi / 2, \pi)$ and positive in the remaining interval $(-\pi / 2, \pi / 2)$,

$$
\begin{aligned}
\int_{-\pi}^{\pi}|\cos x| d x= & \int_{-\pi}^{-\pi / 2}(-\cos x) d x+\int_{-\pi / 2}^{\pi / 2} \cos x d x \\
& +\int_{\pi / 2}^{\pi}(-\cos x) d x=4
\end{aligned}
$$

Therefore, $\left|a_{1}\right| \leqq 4 / \pi$. However, if $f_{1}(x)=-1,-\pi<x<-\pi / 2 ; f_{1}(x)$

${ }^{1}$ Presented to the Society, September 8, 1939.

${ }^{2}$ American Journal of Mathematics, vol. 61 (1939). 
$=1,-\pi / 2<x<\pi / 2 ; f_{1}(x)=-1, \pi / 2<x<\pi ;$ then

$$
\begin{aligned}
\int_{-\pi}^{\pi} f_{1}(x) \cos x d x= & \int_{-\pi}^{-\pi / 2}(-\cos x) d x+\int_{-\pi / 2}^{\pi / 2} \cos x d x \\
& +\int_{\pi / 2}^{\pi}(-\cos x) d x
\end{aligned}
$$

and the bound $4 / \pi$ will actually be attained for the function $f_{1}(x)$.

Likewise, $\int_{-\pi}^{\pi}|\cos n x| d x$ and $\int_{-\pi}^{\pi}|\sin n x| d x$ both equal four, so that $\left|a_{n}\right|$ and $\left|b_{n}\right| \leqq 4 / \pi$; these bounds will be attained for the functions signum $\cos n x$ and signum $\sin n x$ respectively.

But these bounds for the Fourier coefficients cannot be attained simultaneously. If one of the coefficients is too large, the others must be small. For example: $b_{1}$ and $b_{2}$ must satisfy the following inequality:

$$
\left(\frac{\pi}{4} b_{1}\right)^{2}+\frac{\pi}{4}\left|b_{2}\right| \leqq 1
$$

so that if $b_{1}=4 / \pi$ then $b_{2}=0$ while if $b_{2}=4 / \pi$ then $b_{1}=0$. Conversely, any numbers $b_{1}, b_{2}$ satisfying this relation will be the Fourier coefficients of some bounded function.

Similar relations hold between the other Fourier coefficients, but they are much more complicated:

$$
\begin{gathered}
a_{n}^{2}+b_{n}^{2} \leqq\left(\frac{4}{\pi}\right)^{2} \\
\left(\frac{\pi}{4} b_{1}+1\right)^{3}-3\left(\frac{\pi}{4} b_{1}+1\right)-1 \\
\leqq 3\left(\frac{\pi}{4} b_{3}\right) \leqq\left(\frac{\pi}{4} b_{1}-1\right)^{3}-3\left(\frac{\pi}{4} b_{1}-1\right)+1 .
\end{gathered}
$$

After the inequalities for the Fourier coefficients are obtained, they are used to derive an approximation theorem. But instead of being an approximation to the function itself, it will be an approximation to the Fourier development of the function. More precisely: take a bounded measurable function $f(x)$ whose absolute value is less than one; let its Fourier development be $a_{0} / 2+\sum\left(a_{n} \cos n x+b_{n} \sin n x\right)$. Then, for any $N$ there exists a function $g_{N}(x)$ which takes the values 1 and -1 only, such that the first $N$ Fourier coefficients of $g_{N}(x)$ are the same as the first $N$ Fourier coefficients of $f(x)$.

In general, there will be $N+1$ discontinuities. If $f(x)$ is even or odd, $g_{N}(x)$ will be even or odd. For example, let $f(x)=(\pi-x) / \pi$, 
$0<x<\pi ; f(-x)=-f(x)$. Then its Fourier series will be

$$
\begin{aligned}
& \frac{2}{\pi} \sum_{1}^{\infty} \frac{\sin n x}{n} \\
& g_{1}(x)=+1, \quad 0<x<\frac{2 \pi}{3} ; \quad g_{2}(x)=+1, \quad 0<x<\frac{\pi}{2} \\
& g_{1}(x)=-1, \quad \frac{2 \pi}{3}<x<\pi ; \quad g_{2}(x)=-1, \quad \frac{\pi}{2}<x<\frac{2 \pi}{3} \\
& g_{2}(x)=+1, \quad \frac{2 \pi}{3}<x<\pi .
\end{aligned}
$$

Incidentally, one of the inequalities leads to the following result: If $F(x)$ is a bounded measurable function in absolute value less than $\frac{1}{2}$,

$$
\int_{0}^{\pi} F(x) \cos x d x \leqq \cos \left(\int_{0}^{\pi} F(x) d x\right) .
$$

The method used in proving the above inequalities is as follows: Let $f(x)$ be a bounded measurable function in $(-\pi, \pi)$ such that $|f(x)| \leqq 1$. Let the Fourier development of $f(x)$ be

$$
\frac{a_{0}}{2}+\sum\left(a_{n} \cos n x+b_{n} \sin n x\right) \text {. }
$$

Consider the expression

$$
f_{n}(\mu, a) \equiv \mu_{0} a_{0}+\mu_{1} b_{1}+\mu_{2} a_{1}+\cdots+\mu_{2 n-1} b_{n}+\mu_{2 n} a_{n}
$$

where $\mu_{0}, \mu_{1}, \cdots, \mu_{2 n}$ are arbitrary real parameters. The functions $f_{n}(\mu, a)$ can be expressed in terms of the function $f(x)$ by using the usual formulae for Fourier coefficients

$$
a_{n}=\frac{1}{\pi} \int_{-\pi}^{\pi} f(x) \cos n x d x, \quad b_{n}=\frac{1}{\pi} \int_{-\pi}^{\pi} f(x) \sin n x d x .
$$

From these formulae and (1), it is clear that

$$
\begin{aligned}
f_{n}(\mu, a) & =\frac{1}{\pi} \int_{-\pi}^{\pi} f(x)\left[\mu_{0}+\mu_{1} \sin x+\mu_{2} \cos x+\cdots\right. \\
& \left.\quad+\mu_{2 n-1} \sin n x+\mu_{2 n} \cos n x\right] d x \\
& \equiv \frac{1}{\pi} \int_{-\pi}^{\pi} f(x) C_{n}(\mu, x) d x,
\end{aligned}
$$

say. 
Assuming the $\mu$ 's are fixed, the maximum of $f_{n}(\mu, a)$ will be attained when the integrand $f(x) C_{n}(\mu, x)$ is as large as possible. But

$$
\left|f(x) C_{n}(\mu, x)\right| \leqq\left|C_{n}(\mu, x)\right|
$$

since $|f(x)| \leqq 1$. However, if $f(x)$ is taken as signum $C_{n}(\mu, x)$; that is, $f(x)=1$ when $C_{n}(\mu, x)>0$ and $f(x)=-1$ when $C_{n}(\mu, x)<0$, then

$$
\left|f(x) C_{n}(\mu, x)\right|=\left|C_{n}(\mu, x)\right| \text {. }
$$

The following result is now obvious:

$$
f_{n}(\mu, a) \leqq \frac{1}{\pi} \int_{-\pi}^{\pi}\left|C_{n}(\mu, x)\right| d x \equiv D_{n}(\mu),
$$

and the equality holds only when

$$
f(x)=\operatorname{signum} C_{n}(\mu, x) \equiv g_{n}(\mu, x) .
$$

For example, if $n=1$ and $\mu_{1}=0$, formula (2) states that

$$
\mu_{0} a_{0}+\mu_{2} a_{1} \leqq \frac{1}{\pi} \int_{-\pi}^{\pi} f_{1}(x) C_{1}(\mu, x) d x
$$

where

$$
f_{1}(x)=\operatorname{signum}\left(\mu_{0}+\mu_{2} \cos x\right) .
$$

Assume $\mu_{0}+\mu_{2} \cos x=0$ has the real roots $\pm \alpha_{1}$; then $g_{1}(\mu, x)$ will be defined as follows:

and

$$
\begin{aligned}
& g_{1}(\mu, x)=+1, \quad-\alpha_{1}<x<\alpha_{1}, \\
& g_{1}(\mu, x)=-1, \quad\left\{\begin{array}{c}
-\pi<x<-\alpha_{1}, \\
\alpha_{1}<x<\pi, \\
g_{1}(\mu, x)=-1, \quad \text { if } \mu_{0}>0 ; \\
g_{1}(\mu, x)=+1, \quad\left\{\begin{array}{c}
-\pi<x<\alpha_{1}, \\
\alpha_{1}<x<\pi,
\end{array} \text { if } \mu_{0}<0 ;\right.
\end{array}\right.
\end{aligned}
$$

$$
\begin{aligned}
\int_{-\pi}^{\pi}\left|C_{1}(\mu, x)\right| d x & = \pm \frac{1}{\pi} \int_{-\pi}^{\pi} g_{1}(\mu, x) C_{1}(\mu, x) d x \\
= \pm \frac{1}{\pi}\left[\int_{-\pi}^{-\alpha_{1}}-C_{1}(\mu, x) d x+\int_{-\alpha_{1}}^{\alpha_{1}} C_{1}(\mu, x) d x\right. & \left.+\int_{\alpha_{1}}^{\pi}-C_{1}(\mu, x) d x\right]
\end{aligned}
$$




$$
= \pm \frac{1}{\pi}\left\lfloor\mu_{0}(4 \alpha-2 \pi)+\mu_{2} 4 \sin \alpha\right\rfloor= \pm \frac{4}{\pi}\left[\mu_{0}\left(\alpha-\frac{\pi}{2}\right)+\mu_{2} \sin \alpha\right] .
$$

If $\mu_{0}$ is positive, the upper sign holds; if $\mu_{0}$ is negative, the lower sign is used.

But $C_{1}\left(\mu, \alpha_{1}\right)=0$; that is, $\mu_{0}+\mu_{2} \cos \alpha_{1}=0$ or

$$
\begin{aligned}
\cos \alpha & =-\mu_{0} / \mu_{2}, \\
\alpha & =\operatorname{arc} \cos \left(-\mu_{0} / \mu_{2}\right), \quad \alpha-\pi / 2=\arcsin \mu_{0} / \mu_{2}, \\
\sin \alpha & =\left(1-\mu_{0}^{2} / \mu_{2}^{2}\right)^{1 / 2},
\end{aligned}
$$

so that

$$
D_{1}(\mu)= \pm \frac{4}{\pi}\left[\mu_{0} \arcsin \left(\mu_{0} / \mu_{2}\right)+\mu_{2}\left(1-\mu_{0}^{2} / \mu_{2}^{2}\right)^{1 / 2}\right]
$$

Therefore

$$
\mu_{0} a_{0}+\mu_{2} a_{1} \leqq \pm \frac{4}{\pi}\left[\mu_{0} \arcsin \left(\mu_{0} / \mu_{2}\right)+\mu_{2}\left(1-\mu_{0}^{2} / \mu_{2}^{2}\right)^{1 / 2}\right]
$$

where $a_{0}$ and $a_{1}$ are the Fourier coefficients of any bounded function. It is easy to see that the equality sign holds only when $a_{0}$ and $a_{1}$ equal the Fourier coefficients of $g_{1}(\mu, x)$.

Similar results hold for any $n$. Asume $C_{n}(\mu, x)=0$ has $2 n$ real roots $\alpha_{i}$ where $-\pi<\alpha_{1} \leqq \alpha_{2} \leqq \alpha_{3} \leqq \cdots \leqq \alpha_{2 n} \leqq \pi$. Call $-\pi=\alpha_{0}$ and $\pi=\alpha_{2 n+1}$.

Assume $C_{n}(\mu, x)$ is positive between $\alpha_{0}$ and $\alpha_{1}$. Then $g_{n}(\mu, x)$ will be defined as follows: $g_{n}(\mu, x)=(-1)^{i}, \alpha_{i}<x<\alpha_{i+1}, i=0,1, \cdots, 2 n+1$. If $C_{n}(\mu, x)$ is negative, then $g_{n}(\mu, x)=(-1)^{i+1}, \alpha_{i}<x<\alpha_{i+1}$. Therefore, $D_{n}(\mu)=\frac{1}{\pi} \int_{-\pi}^{\pi} g_{n}(\mu, x) C_{n}(\mu, x) d x= \pm \frac{1}{\pi} \sum_{0}^{2 n+1} \int_{\alpha_{1}}^{\alpha_{i+1}}(-1)^{i} C_{n}(\mu, x) d x$ so that from (2)

$$
\mu_{0} a_{0}+\mu_{1} b_{1}+\mu_{2} a_{1}+\cdots+\mu_{2 n-1} b_{n}+\mu_{2 n} a_{n} \leqq D_{n}(\mu) .
$$

Let

$$
a_{i}=x_{2 i}, \quad b_{i}=x_{2 i-1} .
$$

Consider the $2 n+1$ dimensional space of points whose coordinates are the $x_{j}(j=0,1,2, \cdots, 2 n+1)$. The inequality (4) can be written as

$$
\sum_{j=0}^{2 n+1} \mu_{j} x_{j} \leqq D_{n}(\mu)
$$


This implies that, for any bounded measurable function, the point $P$ whose coordinates are given by (5) must lie on one side of the hyperplane whose equation is

$$
\sum_{0}^{2 n+1} \mu_{j} x_{j}=D_{n}(\mu)
$$

The point $P$ will lie on the plane if (4) becomes an equality. This will occur when and only when $f(x)$ has its first $2 n+1$ Fourier coefficients equal to those of $g_{n}(\mu, x)$.

Since the $\mu_{j}$ were arbitrary, the same result holds for any set $\mu_{0}, \mu_{1}, \cdots, \mu_{2 n+1}$. Therefore, the point $P$, whose coordinates are $\left(x_{j}\right)$, $j=0,1, \cdots, 2 n+1$, must lie on one side of every plane in the family of planes defined by the equation (6).

The previous example will clarify this. Equation (3) implies that if, for any $f(x), a_{0}$ is plotted as the $x$ coordinate and $a_{1}$ as the $y$ coordinate of a point, then such points will lie on the origin side of the straight line whose equation is

$$
\mu_{0} x+\mu_{2} y=D_{1}(\mu) .
$$

The point will lie on the straight line if and only if $f(x)$ has its Fourier coefficients $a_{0}$ and $a_{1}$ equal to those of $g_{1}(\mu, x)$.

Since the $\mu$ 's were arbitrary, the same result holds for any set $\mu_{0}, \mu_{2}$. Therefore, the point $P$, whose coordinates are $\left(a_{0}, a_{1}\right)$ must lie on one side of every line in the family of straight lines defined by the equation

$$
\mu_{0} x+\mu_{2} y=D_{1}(\mu) .
$$

This implies that the point $P$ will lie inside the region enveloped by these straight lines.

The usual procedure in finding the equation of this envelope is, first, to differentiate partially with respect to the parameter, and second, to eliminate the parameters between the resulting equation and the original equation. The partial differentiation results in the following two equations:

$x=\frac{\partial D}{\partial \mu_{0}}= \pm(4 / \pi) \arcsin \left(\mu_{0} / \mu_{2}\right), \quad y=\frac{\partial D}{\partial \mu_{2}}= \pm(4 / \pi)\left(1-\mu_{0}^{2} / \mu_{2}^{2}\right)^{1 / 2}$.

After the elimination of the parameters, the following equation is obtained for the envelope:

$$
y=(4 / \pi) \cos (\pi x / 4) .
$$

This implies that 


$$
a_{1} \leqq(4 / \pi) \cos \left(\pi a_{0} / 4\right)
$$

Equality will hold, as before, if $f(x)$ has the same first two Fourier coefficients as any function $g_{1}(\mu, x)$.

In the general case, the equation of the envelope is obtained by the same method. When formula (6) is differentiated partially with respect to the parameters, the following equations result:

$$
x_{j}=\frac{\partial D_{n}(\mu)}{\partial \mu_{j}}, \quad j=0,1, \cdots, 2 n+1 .
$$

But

$$
\frac{\partial D_{n}(\mu)}{\partial \mu_{j}}= \pm \frac{1}{\pi} \frac{\partial}{\partial \mu_{j}}\left[\sum_{i=0}^{2 n+1} \int_{\alpha_{i}}^{\alpha_{i+1}}(-1)^{i} C_{n}(\mu, x) d x\right]
$$

where the $\alpha_{i}$, the roots of $C_{n}(\mu, x)=0$, also depend on the $\mu_{j}$. Now

$$
\begin{aligned}
& \frac{\partial}{\partial \mu_{j}} \int_{\alpha_{i}}^{\alpha_{i+1}}(-1)^{i} C_{n}(\mu, x) d x=\int_{\alpha_{i}}^{\alpha_{i+1}}(-1)^{i} \frac{\partial C_{n}(\mu, x)}{\partial \mu_{j}} d x \\
&+(-1)^{i} C_{n}\left(\mu, \alpha_{i+1}\right) \frac{\partial \alpha_{i+1}}{\partial \mu_{j}}-(-1)^{i} C_{n}\left(\mu, \alpha_{i}\right) \frac{\partial \alpha_{i}}{\partial u_{j}}
\end{aligned}
$$

by the standard rule for differentiating under the integral sign.

However $C_{n}\left(\mu, \alpha_{i+1}\right)=C_{n}\left(\mu, \alpha_{i}\right)=0$ and

$$
\begin{aligned}
\frac{\partial C_{n}(\mu, x)}{\partial \mu_{j}} & =\sin (j+1) x / 2 \text { if } j \text { is odd, } \\
& =\cos (j x / 2) \text { if } j \text { is even, }
\end{aligned}
$$

so that

$$
\begin{aligned}
x_{j} & = \pm \frac{1}{\pi} \sum_{i=0}^{2 n+1} \int_{\alpha_{i}}^{\alpha_{i+1}}(-1)^{i} \sin (j+1) x / 2 d x \text { if } j \text { is odd } \\
& = \pm \frac{1}{\pi} \sum_{i=0}^{2 n+1} \int_{\alpha_{i}}^{\alpha_{i+1}}(-1)^{i} \cos (j x / 2) d x \text { if } j \text { is even, }
\end{aligned}
$$

where the signs are either all positive or all negative.

Instead of eliminating the parameters $\mu$, it is simpler to eliminate the parameters $\alpha$, thus obtaining the equation of the envelope $\phi_{n}\left(x_{j}\right)=0$ which implies the inequality

$$
\phi_{n}(a, b) \leqq 0 .
$$

Since $\phi_{n}\left(x_{j}\right)$ is obtained as the envelope of planes, it is obvious that 
the region bounded by it is the convex hull of a family of half-spaces. Again, the inequality will hold if and only if $f(x)$ has its first $2 n+1$ Fourier coefficients $\left(a_{0}, a_{1}, b_{1}, \cdots, a_{n}, b_{n}\right)$ equal to the corresponding Fourier coefficients of any one of the functions $g_{n}(\mu, x)$.

A few illustrations will clarify this method. Let $n=2$ and $\mu_{0}=\mu_{2}$ $=\mu_{4}=0$. Then the $\alpha_{i}$ will be the roots of the equation

$$
\mu_{1} \sin x+\mu_{3} \sin 2 x=0,
$$

and from formula $(7)$

$$
x_{1}= \pm \frac{1}{\pi} \sum \int_{\alpha_{i}}^{\alpha_{i+1}}(-1)^{i} \sin x d x= \pm\left(-4 / \pi \cos \alpha_{3}\right)
$$

since $\alpha_{1}=-\alpha_{3}$, and

$$
x_{i}= \pm \frac{1}{\pi} \sum \int_{\alpha_{i}}^{\alpha_{i+1}}(-1)^{i} \sin 2 x d x= \pm(2 / \pi)\left(1-\cos 2 \alpha_{3}\right) .
$$

Therefore $\left(\pi x_{1} / 4\right)^{2}+\pi x_{3} / 4=1$ or $\left(\pi x_{1} / 4\right)^{2}-\pi x_{3} / 4=1$ according as the plus or minus signs are used. But since $x_{1}$ was the coordinate for $b_{1}$ and $x_{3}$ for $b_{2}$, the following inequality results:

$$
\left(\pi b_{1} / 4\right)^{2}+\pi\left|b_{3}\right| / 4 \leqq 1 \text {. }
$$

From the result proved above: that the Fourier coefficients of $f(x)$ satisfy certain inequalities or, stating it geometrically, lie in certain convex regions, the approximation theorem follows. The theorem can be stated as follows:

Let $f(x)$ be a real, bounded, measurable function in absolute value less than one. Let its Fourier development be

$$
\frac{a_{0}}{2}+\sum\left(a_{n} \cos n x+b_{n} \sin n x\right) \text {. }
$$

Then for every $N$, there exists a step-function $g_{N}(x)$ which takes on the values of +1 and -1 only, such that the first $N$ Fourier coefficients of $g_{N}(x)$ are the same as the first $N$ Fourier coefficients of $f(x)$, that is,

$$
\begin{aligned}
a_{n}=\frac{1}{\pi} \int_{-\pi}^{\pi} g_{N}(x) \cos n x d x, \quad b_{n}=\frac{1}{\pi} \int_{-\pi}^{\pi} g_{N}(x) \sin n x d x, \\
n=0,1, \cdots, N .
\end{aligned}
$$

Again, let $a_{i}=x_{2 i}^{\prime}, b_{i}=x_{2 i-1}^{\prime}$. Then, as before, the point $P^{\prime}$ whose coordinates are $\left(x_{0}^{\prime}, x_{1}^{\prime}, \cdots, x_{2 N}^{\prime}\right)$ must satisfy the inequality (8): 
$\phi_{N}\left(x_{j}^{\prime}\right) \leqq 0$, or, geometrically, the point $P^{\prime}$ must lie inside the convex region bounded by the hypersurface whose equation is

$$
\phi_{N}\left(x_{0}, x_{1}, x_{2}, \cdots, x_{2 N}^{\prime}\right)=0 .
$$

Suppose now that $P^{\prime}$ lies on the hypersurface, that is, its coordinates satisfy the equation

$$
\phi_{N}\left(x_{0}^{\prime}, x_{1}^{\prime}, x_{2}^{\prime}, \cdots, x_{2 N}^{\prime}\right)=0 .
$$

Since $\phi_{N}\left(x_{j}\right)=0$ was obtained as the envelope of the family of hyperplanes represented by equation (6), $\sum \mu_{j} x_{j}=D_{N}(\mu)$, there will be a plane of the family tangent to the surface at $P^{\prime}$. But since $P^{\prime}$ lies on this plane, whose equation can be written

$$
\mu_{0}^{\prime} x_{0}+\mu_{1}^{\prime} x_{1}+\mu_{2}^{\prime} x_{2}+\cdots+\mu_{2 N}^{\prime} x_{2 N}=D_{N}\left(\mu^{\prime}\right),
$$

its coordinates must equal those of the function $g_{N}\left(\mu^{\prime}, x\right)$. Therefore, $g_{N}\left(\mu_{0}^{\prime}, \cdots, \mu_{2 N}^{\prime}, x\right)$ is the function described in the theorem.

If $P^{\prime}$ lies inside the hypersurface, consider the points in $2 N+3$ dimensional space whose coordinates are $\left(x_{0}^{\prime}, x_{1}^{\prime}, x_{2}^{\prime}, \cdots, x_{2 N}^{\prime}, x_{2 N+1}\right.$, $\left.x_{2 N+3}\right)$ where $x_{2 N+1}$ is an arbitrary variable. They will lie on the straight line through $\left(x_{0}^{\prime}, x_{1}^{\prime}, x_{2}^{\prime}, \cdots, x_{2 N}^{\prime}, 0,0\right)$ perpendicular to the hyperplane $x_{2 N+1}=x_{2 N+2}=0$.

Since $\phi_{N+1}\left(x_{j}\right)=0$ is a closed convex surface for all $n$, the above straight line will intersect it in some point whose coordinates are $\left(x_{0}^{\prime}, x_{1}^{\prime}, x_{2}^{\prime}, \cdots, x_{2 N}^{\prime}, x_{2 N+1}^{\prime}, x_{2 N+2}^{\prime}\right)$. Just as before, there will exist a step-function $g_{N+1}(x)$ whose Fourier development is $a_{0}^{\prime} / 2$ $+\sum\left(a_{n}^{\prime} \cos n x+b_{n}^{\prime} \sin n x\right)$ and such that $a_{i}^{\prime}=x_{2 i}^{\prime}, b_{i}^{\prime}=x_{2 i-1}^{\prime} ; i=0,1$, $2, \cdots, N+1$.

This function satisfies the conditions of the theorem for

$$
x_{2 i}^{\prime}=a_{i}=a_{i}^{\prime}, \quad x_{2 i-1}^{\prime}=b_{i}^{\prime}=b_{i} .
$$

UNIVERSITY OF Wisconsin 